\title{
Adaptive Fuzzy High-Order Super-Twisting Sliding Mode Controller for Uncertain Robotic Manipulator
}

DOI 10.1515/jisys-2016-0020

Received March 16, 2016; previously published online October 4, 2016.

\begin{abstract}
This paper presents a novel adaptive fuzzy high-order super-twisting sliding mode controller, based on the modified super-twisting control (STC), to achieve accurate trajectory tracking for a robotic manipulator with unknown structured uncertainties, parametric uncertainties, and time-varying external disturbances. Initially, a non-linear homogeneous sliding manifold is designed to achieve finite-time convergence, better robustness, and good transient characteristics. Afterwards, conventional STC is modified with the new sliding surface that eliminates the limitation of STC application only on relative degree 1 systems. Moreover, two adaptive fuzzy systems are designed to replace the STC signals for handling the chattering problem and overestimating the controller gains. These fuzzy systems are continuously adjusted by two adaptation laws that are deduced from the Lyapunov stability theory. These adaptive laws need only a sliding surface variable as an input and generate the optimal controller gains as an output. The finite-time convergence and stability of the proposed controller is analyzed by the homogeneous Lyapunov stability theory. Finally, to show the efficacy of the proposed method, the controller is simulated on a 2-degree-of-freedom planar robotic manipulator to obtain the accurate trajectory tracking. Simulation results demonstrate the superiority of the proposed control scheme in the presence of structured and unstructured uncertainties.
\end{abstract}

Keywords: Adaptive tuning, finite time stability, fuzzy logic control, high-order sliding mode control, robotic manipulator, super-twisting algorithm, uncertainty.

\section{Introduction}

Industrial robotic manipulators (RMs) are designed to perform various sophisticated tasks, such as drilling, welding, assembly, painting, etc., with high accuracy as a prerequisite [8]. However, designing a highperformance controller for RM is very difficult due to the inherent presence of uncertainties and disturbances such as payload variation, unknown disturbances, Coulomb friction, backlash, dead zone, time-varying friction, cross-coupling effect, etc. [7]. These uncertainties, if not perfectly compensated, could adversely affect the desired control performance and positioning accuracy, which could further lead to system instability [46]. Therefore, to achieve high-trajectory tracking accuracy and faster convergence, an effective and intelligent control scheme is essentially required.

Many elegant control techniques, such as decentralized control [26], feedback linearization control [39], adaptive control [29,35], robust control [34], adaptive robust control [44], observer-based control [11], and sliding mode control (SMC) [41, 42], etc., have been proposed and widely applied for stabilizing RM systems. Although these control strategies have shown their effectiveness in achieving the desired performance, the quench of obtaining intelligent robotic control led toward soft computing paradigms in recent years [20].

Among aforementioned control methods, sliding mode controllers are popular and the most widely used method because of their simple control design and robustness against both system parameter uncertainties

*Corresponding author: Ankur Goel, Department of Electrical Engineering, National Institute of Technology Kurukshetra, Kurukshetra, Haryana, India, e-mail: anckurgoel@gmail.com

Akhilesh Swarup: Department of Electrical Engineering, National Institute of Technology Kurukshetra, Kurukshetra, Haryana, India 
and external disturbances [30, 41]. Nevertheless, the switching control which handles system uncertainties, requires an infinite switching on the sliding surface. Additionally, actuator dynamics, signal delays, and other factors helped in the generation of undesired chattering phenomenon. Moreover, the upper bounds of the external disturbances and the structural uncertainties must be well known to obtain a stable closed loop system. Another issue in conventional SMC is that of asymptotic convergence, which, in fact, is not suitable for precise robotic tracking performance [10]. In order to obliterate chattering, many momentous methods, such as boundary layer method, modified switching signal with saturation, etc. [42], have been introduced; however, the presence of the finite steady-state error may cause degradation of the tracking ability. Therefore, to improve the performance of the classical SMC, the high-order sliding mode (HOSMC) control approach has been developed [24]. In HOSMC, the designed sliding variable $s$ has relative degree greater than 1 with respect to the control. Then, the discontinuous control action is applied on the higher derivative of $s$ to start the sliding motion on $s=0$, which provides an effective solution to the chattering problem. The HOSMC shows better robustness against different uncertainties, and provides a higher-order precision and finite-time convergence of the states. The twisting algorithm, super-twisting control (STC), integral SMC (I-SMC), terminal sliding mode (TSM) [43] and its modified versions [2, 6], etc., are popular HOSMC control methods. However, the common drawback of these methods is the proper selection of the controller gains, essential to obtain finite-time convergence feature. Moreover, very few HOSMC applications have been reported in the area of robotic control systems [5, 15, 47], and hence there is a need to analyze the HOSMC for RM to obtain intelligent and effective control systems.

In recent years, fuzzy logic has been widely applied successfully to enhance the efficiency of SMC in controlling uncertain non-linear systems [37]. So far, a large number of fuzzy SMC algorithms (FLSMCs) have been developed for robot control systems [12, 17, 25, 45]. The state of the art of classical SMC with soft computing has been surveyed in Refs. [21, 32]. In Ref. [31], the friction phenomenon has been considered for setpoint tracking of robot manipulator, and stability is proved by Lyapunov theorem and Lasalle's theorem. In Ref. [9], a hybrid approach of fuzzy logic controller and SMC has been given to compute equivalent control effort. The performance of RM has been enhanced by continuous computation of sliding parameters in Ref. [19]. Authors in Ref. [38] combine fuzzy logic and SMC to overcome uncertainties and disturbances during trajectory tracking of the RM. In Refs. [22, 36], evolutionary optimization techniques are used for optimizing control coefficients of the fuzzy sliding mode system. However, these controllers lack mathematical analysis and closed-loop stability analysis. The applications of FLSMC has been broadly classified into three categories [40], viz. adaptive fuzzy method to strengthen the SMC capabilities of disturbance rejection, fuzzy logic-based tuning of controller gain to alleviate chattering, and replacing discontinuous signum function by fuzzy logic in the reaching phase to eliminate the chattering. However, proper selection of the initial values in adaptive fuzzy system is highly essential for fast convergence of the adaptive laws. In Ref. [3], the adaptive fuzzy sliding mode controller (AFSMC) has been proposed to estimate the unknown functions required to overcome the existing uncertainties. However, this controller has a very complex design and may pose some problems during practical implementation.

This paper proposes a robust adaptive fuzzy tracking controller based on the high-order super-twisting SMC (AF-HOSTSMC) for a class of non-linear systems. At first, super-twisting controller, a member of the Lipschitz continuous controller family [23], is modified with a non-linear homogeneous sliding surface to obtain finite-time convergence. Then, to avoid the conservative approach of the gain overestimation process, switching terms of proposed controller are substituted by two adaptive fuzzy systems. This proposed adaptive fuzzy design tries to maintain the originality of the proposed control algorithm and gives the solution to calculate the optimal values of the gains. These novel adaptive systems have single input of the sliding surface and single output of the optimal controller gains. Their updates are performed with the help of two adaptation laws deduced from the Lyapunov stability analysis. The proposed controller is designed to achieve the following performance objectives: (a) faster and finite tracking error convergence to zero in the presence of large uncertainties and external disturbances - a necessary condition for precise motion of RMs; (b) elimination of the undesired chattering phenomenon; (c) less and continuous control input signals - a requirement for better performance of actuators; (d) increased tracking accuracy, due to typical third-order-type controller 
performance obtained by using only two states; (d) model-free control - as upper bounds of the disturbances and uncertainties are not required; and (e) applicable to a wider range of high-order multi-input and multioutput (MIMO) uncertain non-linear systems.

The rest of this article is organized as follows. Mathematical modeling of RM and control objective is given in Section 2. In Section 3, relevant preliminaries and definitions are reviewed and a control problem is formulated. The design of the proposed controller, stability analysis, and finite-time convergence proof are presented in Section 4. In Section 5, the performance of the proposed control method and a few other methods are compared using a 2-degree-of-freedom (2-DoF) planar RM as numerical simulation. Finally, some conclusions drawn from the results are listed in Section 6.

\section{Mathematical Model and Control Objective}

\subsection{Robot System Dynamic Model}

The dynamic mathematical model of uncertain $n$-DoF RM is represented as [39]

$$
M(q) \ddot{q}+C(q, \dot{q}) \dot{q}+G(q)+F(q, \dot{q})=\tau+\tau_{d},
$$

where $q, \dot{q}, \ddot{q} \in R^{n}$ denote the vectors of joint positions, velocities, and accelerations, respectively; $M(q) \in R^{n \times n}$ is a symmetric, positive-definite inertia matrix; $C(q, \dot{q}) \in R^{n \times n}$ expresses the centripetal Coriolis matrix; $G(q) \in R^{n}$ denotes gravitational vector; $F(q, \dot{q}) \in R^{n}$ is the unstructured uncertainties including friction and other disturbances; and $\tau$ and $\tau_{d} \in R^{n}$ denote joint input torque and external disturbances, respectively. Practically, these parameters are difficult to measure precisely due to unavoidable modeling error, payload variations, and other unknown factors. Consequently, the dynamic model [Eq. (1)] is considered as an uncertain model. Therefore, actual values of these parameters can be denoted as $M(q)=M_{0}(q)+\Delta M(q) ; C(q, \dot{q})=C_{0}(q, \dot{q})+\Delta C(q, \dot{q}) ; G(q)=G_{0}(q)+\Delta G(q)$, where the notations $M_{0}(q)$, $C_{0}(q, \dot{q}), G_{0}(q)$ are the nominal matrices, and $\Delta M(q), \Delta C(q, \dot{q}), \Delta G(q)$ denote the system perturbations.

Then, the dynamic manipulator equation [Eq. (1)] can be rewritten as

$$
M_{0}(q) \ddot{q}+C_{0}(q, \dot{q}) \dot{q}+G_{0}(q)=\tau+\tau_{d}+F_{d}(q, \dot{q}, \ddot{q}),
$$

where $F_{d}(q, \dot{q}, \ddot{q})=-\Delta M(q) \ddot{q}-\Delta C(q, \dot{q}) \dot{q}-\Delta G(q)-F(q, \dot{q}) \in R^{n}$ is the total system uncertainty that includes friction and other disturbances. For convenience, the following assumptions are considered about the robot dynamics [47].

Assumption 2.1: Inertia matrix $M(q)$ is bounded for all q even in presence of uncertainty.

Assumption 2.2: $(\dot{M}(q)-2 C(q, \dot{q}))$ is skew symmetric matrix.

Assumption 2.3: The external disturbances $\tau_{d}$ are bounded and unknown but Lipschitz continuous. Also, system modeling uncertainty $F_{d}(q, \dot{q}, \ddot{q})$ is assumed to be bounded.

\subsection{Control Objective}

The control objective is to design a robust finite-time control law that ensures Eq. (3), in spite of external disturbances and uncertainties.

$$
\lim _{t \rightarrow t_{f}}\|e\|=\lim _{t \rightarrow t_{f}}\left\|\left(q-q_{d}\right)\right\|=0,
$$

where $q_{d} \in R^{n}$ is a double differentiable desired trajectory, $e=\left(q-q_{d}\right)$ is trajectory tracking error, and $t_{f}$ is finite time. 


\section{Preliminaries and Problem Formulation}

This section reviews some notations and definitions that are relevant to this work.

\subsection{Preliminaries}

\subsubsection{Notation}

For a real variable $a \in R$ to a real power $b \in R$, then $\lfloor a]^{b}=|a|^{b} \operatorname{sgn}(a)$, e.g. $\left\lfloor\left. z\right|^{2}=|z|^{2} \operatorname{sgn}(z) \neq z^{2}\right.$ [4]. If $p$ is an odd number, then $\lfloor z\rceil^{p}=|z|^{p}$ e.g. $\lfloor z\rceil^{0}=\operatorname{sgn}(z),\lfloor z\rceil^{0} z^{p}=|z|^{p}$.

\subsubsection{Finite Time Convergence}

Consider a closed-loop system represented as [4]

$$
\dot{x}(t)=u(t) ; x\left(t_{0}\right)=x_{0},
$$

where $x(t) \in R$ is a system state and $u(t) \in R$ is a control input.

Definition 3.1 ([1]): The state of the system Eq. (4), i.e. trajectory $x(t) \in R$, is global finite-time convergent to a point $x_{f} \in R^{n}$ if for any initial conditions $x\left(t_{0}\right)=x_{0} \in R^{n}$ there exists a time moment $T\left(x_{0}\right)<\infty$ s.t. $x(t)=x_{f}$ for all $t \geq t_{f}$

Definition 3.2: ([1]) For the system Eq. (4), suppose there exists a continuous positive definite function $V: R^{n} \rightarrow R$, real numbers $\beta>0, \gamma \in(0,1)$, and an open neighborhood $U_{0} \subseteq R^{n}$ of the origin such that the following inequalities are satisfied:

$$
\dot{V}(x)+\beta(V(x))^{\gamma} \leq 0, x \in U_{0} \backslash\{0\} .
$$

Then, the origin is a finite-time-stable equilibrium of Eq. (4). Moreover, if $T$ is the settling-time function, then

$$
T(x) \leq \frac{1}{\beta(1-\gamma)} V(x)^{1-\gamma} .
$$

If, in addition, $U_{0}=R^{n}, V$ is proper, and $\dot{V}$ is negative definite, then the origin is a globally finite-time-stable equilibrium of Eq. (4).

\subsection{Problem Formulation}

The problem of robust finite-time trajectory tracking of the manipulator is initiated with the design of sliding surface $\sigma \in R^{n}$ such that

$$
\sigma=e=\left(q-q_{d}\right) .
$$

Clearly, the second time derivative of Eq. (7) is represented as

$$
\begin{aligned}
\ddot{\sigma} & =\ddot{e}=\left(\ddot{q}-\ddot{q}_{d}\right) \\
& =M_{0}^{-1}(q)\left[\tau+\tau_{d}+F_{d}(q, \dot{q}, \ddot{q})-C_{0}(q, \dot{q}) \dot{q}-G_{0}(q)\right]-\ddot{q}_{d} \\
& =M_{0}^{-1}(q) \tau+M_{0}^{-1}(q)\left[\tau_{d}+F_{d}(q, \dot{q}, \ddot{q})\right]-M_{0}^{-1}(q)\left[C_{0}(q, \dot{q}) \dot{q}+G_{0}(q)\right]-\ddot{q}_{d} \\
& =-M_{0}^{-1}(q)\left[C_{0}(q, \dot{q}) \dot{q}+G_{0}(q)\right]-\ddot{q}_{d}+M_{0}^{-1}(q) \tau+\tilde{d},
\end{aligned}
$$


where $\tilde{d}=M_{0}^{-1}(q)\left[\tau_{d}+F_{d}(q, \dot{q}, \ddot{q})\right] \in R^{n}$ is unknown, Lipschitz continuous, lumped uncertainty, such that $|\dot{\tilde{d}}| \leq \rho, \rho>0$.

Remark 3.3: Equation (8) could be represented as differential inclusion $\ddot{\sigma} \in[-C, C]+\left[K_{m}, K_{M}\right] u$, where bounds of constants $C, K_{m}, K_{M}$ are state dependent (for more details, see Ref. [23]).

Remark 3.4: The relative degree $r$ of the system Eq. (2) with respect to Eq. (8) is 2, and remains constant even in presence of disturbance term $\tilde{d}$.

Considering new system variables $\xi_{1}, \xi_{2} \in R^{n}$, the sliding vector dynamics could be written as

$$
\begin{aligned}
\dot{\xi}_{1}=\dot{\sigma} & =\xi_{2} \\
\dot{\xi}_{2}=\ddot{\sigma} & =-M_{0}^{-1}(q)\left[C_{0}(q, \dot{q}) \dot{q}+G_{0}(q)\right]-\ddot{q}_{d}+M_{0}^{-1}(q) \tau+\tilde{d} \\
& =H_{\text {nom }}(t, q)+G_{\text {nom }}(t, q) \tau+\tilde{d},
\end{aligned}
$$

where $H_{\text {nom }}(t, q)=-M_{0}^{-1}(q)\left[C_{0}(q, \dot{q}) \dot{q}+G_{0}(q)\right]-\ddot{q}_{d}$ and $G_{\text {nom }}(t, q)=M_{0}^{-1}$. Under nominal condition, i.e. $(\tilde{d}=0)$, the system Eq. (9) will have following feedback control:

$$
\tau=G_{\text {nom }}^{-1}(t, q)\left(-H_{\text {nom }}(t, q)+u(t)\right),
$$

where $u \in R^{n}$ is an auxiliary input. Hence, under lumped uncertainty condition, using Eqs. (9) and (10), we get

$$
\begin{aligned}
& \dot{\xi}_{1}=\xi_{2} \\
& \dot{\xi}_{2}=u(t)+\tilde{d} .
\end{aligned}
$$

Finally, the control objective is modified to design the effective auxiliary control input $u(t)$, so that states $\left(\xi_{1}\right.$, $\xi_{2}$ ) of the system Eq. (11) converge to their respective equilibrium in finite time under the presence of uncertainty and external disturbances.

\section{The Controller Design}

In the previous subsection, the trajectory tracking control problem is formulated as the double integrator system. Obviously, the above-mentioned objective can be robustly achieved by the classical SMC; however, the requirement of the upper bounds of the disturbances and the asymptotic state convergence restrict its application to robotic control problems.

Consequently, to ensure the robustness and finite-time state convergence, we propose here to modify the conventional super-twisting controller with a geometric homogeneity concept, which will provide the performance of the third-order sliding mode controller virtually. Indeed, the STC will contribute to the global robustness, and the third-order sliding mode will be instrumental into chattering alleviation and finite-time convergence.

Inspired by the above discussion, in the following subsection, conventional STC is reviewed initially and afterwards the design of the proposed controller is elaborated.

\subsection{Conventional Super-Twisting Controller}

The super-twisting controller is a popular Lipschitz continuous second-order sliding mode controller. It requires a system with dynamics having relative degree 1 with respect to sliding variable " $s$ " [13], i.e.

$$
\dot{s}=h(t, x, u)+g(t, x) u(t),
$$

where $h(t, x, u)$ and $g(t, x)$ have known boundaries such that $\xi \geq|h(t, x, u)|, \xi>0$ and $0<K_{m} \leq g(t, x) \leq K_{M}$. 
According to the STC algorithm, the control input is represented as

$$
\begin{gathered}
u(t)=-\lambda|s|^{\gamma} \operatorname{sign}(s)+u_{1} \\
\dot{u}_{1}=\left\{\begin{array}{cc}
-u, & |u|>1, \\
-\alpha \operatorname{sign}(s), & |u| \leq 1
\end{array}\right.
\end{gathered}
$$

where $\lambda$ and $\alpha$ are the controller gains. Moreover, to obtain finite-time convergence of the states, the following gain conditions should be satisfied:

$$
\alpha>\frac{\zeta}{K_{m}} ; \quad \lambda^{2} \geq \frac{\xi K_{M}(\alpha+\zeta)}{K_{m}^{2} K_{M}(\alpha-\zeta)} ; \quad 0<\gamma \leq 0.5
$$

Remark 4.1: Generally, practical systems are modeled as second- or higher-order systems, and hence, to apply STC, first-order sliding variable has to be designed with linear combination of the states, which results in asymptotic convergence instead of finite-time convergence.

\subsection{High-Order Super-Twisting SMC Design}

In view of the aforementioned discussion, to design the high-order (third-order) super-twisting controller, the sliding manifold $s_{1} \in R^{n}$ for the system Eq. (11), using the geometric homogeneity theory, is selected as

$$
\left.S_{1}=\xi_{2}+K_{3} L_{1}\right]^{2 / 3} \text {. }
$$

Now, with new sliding manifold [Eq. (15)], the STC control [Eq. (13)] is given as

where

$$
\begin{aligned}
u_{s t}(t) & =-u_{s t}^{a}-u_{s t}^{b} \\
u_{s t}^{a} & =\tilde{K}_{1}\left|s_{1}\right|^{1 / 2} \operatorname{sign}\left(s_{1}\right)
\end{aligned}
$$

$$
\dot{u}_{s t}^{b}=\left\{\begin{array}{cc}
u, & |u|>U_{M} \\
\tilde{K}_{2} \operatorname{sign}\left(s_{1}\right) & |u| \leq U_{M}
\end{array} .\right.
$$

Here, $\tilde{K}_{1}>0$ and $\tilde{K}_{2}>0$ are the controller gains and $K_{3}>0$ is a fixed positive constant used to adjust the slope of the sliding surface.

Remark 4.2: $s_{1}$ [Eq. (15)] is a non-linear, monotone, continuous increasing function, and its equilibrium point is globally finite-time stable [16].

Remark 4.3: Controller [Eq. (16)] is homogeneous of degree $\delta_{f}=-1$ with weights $\alpha=[3,2,1]$ and its solution is understood in the Filippov sense.

Note that in a practical environment, lumped uncertain term $\tilde{d}$ effects could not be ignored, and hence, $u_{s t}$ alone is not sufficient to ensure the desired performance. Therefore, an additional control input component $u_{\text {aux }}$ is added to Eq. (16). The main role of $u_{a u x}$ is to maintain the variables on the sliding surface in the presence of the lumped uncertainty [42]. Therefore, the final proposed control structure for the system Eq. (11) is

$$
u(t)=u_{s t}(t)+u_{a u x}(t)
$$

where

$$
\begin{aligned}
u_{s t}(t) & =-\tilde{K}_{1}\left|s_{1}\right|^{1 / 2} \operatorname{sign}\left(s_{1}\right)-\int_{0}^{t} \tilde{K}_{2} \operatorname{sign}\left(s_{1}\right) d t \\
u_{\text {aux }}(t) & =-\frac{2}{3} K_{3} \xi_{2}\left|\xi_{1}\right|^{-1 / 3} .
\end{aligned}
$$


Consequently, the overall proposed feedback control law [Eq. (10)] structure is

$$
\tau=G_{\text {nom }}^{-1}(t, q)\left\{-H_{\text {nom }}(t, q)-\tilde{K}_{1}\left|s_{1}\right|^{1 / 2} \operatorname{sign}\left(s_{1}\right)-\int_{0}^{t} \tilde{K}_{2} \operatorname{sign}\left(s_{1}\right) d t-\frac{2}{3} K_{3} \xi_{2}\left|\xi_{1}\right|^{-1 / 3}\right\} .
$$

Remark 4.4: The gains $\tilde{K}_{1}$ and $\tilde{K}_{2}$ play an important role in the chattering reduction and in convergence speed control. Generally, in conventional finite-time SMC designs, both $\tilde{K}_{1}$ and $\tilde{K}_{2}$ are invariable, and are linked with the upper bounds of disturbances and uncertainties.

\subsection{Design of Fuzzy Logic-Based Adaptive Laws}

In this section, the design methodology for the selection of controller gains $\tilde{K}_{1}$ and $\tilde{K}_{2}$ is presented. Indeed, in the steady state, i.e. $s_{1}=0$, HOSMC will provide finite-time convergence as per the theory of homogeneity. However, during reaching phase, for optimal selection of gains, knowledge of the upper bounds of the disturbances is essential. Unfortunately, this information is often unavailable practically and leads to overestimation, which further generates undesired high-frequency chattering. Nevertheless, even if this information is available, the functions $\left|s_{1}\right|^{1 / 2}$ and $\operatorname{sign}\left(s_{1}\right)$ in the control design will become major source of chattering. Therefore, there is a need to have a trade-off between the desired performance specification and compatible control action.

To this end, for smooth control output, an intelligent and adaptive methodology is designed that will replace the following switching control functions of the proposed controller [Eq. (16)], i.e.

$$
u_{s t}^{a}=\tilde{K}_{1}\left|s_{1}\right|^{1 / 2} \operatorname{sign}\left(s_{1}\right) \text { and } u_{s t}^{b}=\int_{0}^{t} \tilde{K}_{2} \operatorname{sign}\left(s_{1}\right) d t \text {, }
$$

by two individual fuzzy logic systems (FLSs).

The steps involved in the design of the novel fuzzy logic-based adaptive methodology are as follows:

(a) Step 1: Design of the FLS

Consider a singleton fuzzifier, product reasoning, and center of gravity average defuzzifier with collection of IF-THEN rules in the following form [27]:

$$
\text { Rule } R^{i} \text { : IF } a_{1} \text { is } \bar{F}_{1}^{i} \text { and } \ldots \text { and } a_{n} \text { is } \bar{F}_{n}^{i} \text { THEN } y \text { is } \bar{G}^{i}
$$

where $\bar{F}_{j}^{i}$ and $\bar{G}^{i}$ are fuzzy variables sets with $(j=1,2, \ldots, n), n$ is total number of variables, $(i=1,2$, $\ldots, N$ ), and $N$ is the total number of IF-THEN rules in the rule base. The output $y$ of the fuzzy system is given as

$$
y=\frac{\sum_{i=1}^{N} \theta_{i} \prod_{j=1}^{n} \mu_{A_{j}^{i}}\left(x_{j}\right)}{\sum_{i=1}^{N} \prod_{j=1}^{n} \mu_{A_{j}^{i}}\left(x_{j}\right)}=\psi^{T} \theta,
$$

where $\mu_{A_{j}^{i}}\left(x_{j}\right)$ is membership function of $\left(x_{j}\right)$;

vector $\psi=\left[\psi_{1}(x) \psi_{2}(x) \ldots \psi_{N}(x)\right]^{T}$, where $\psi_{i}(x)=\frac{\prod_{j=1}^{n} \mu_{A_{j}^{i}}\left(x_{j}\right)}{\sum_{i=1}^{N} \prod_{j=1}^{n} \mu_{A_{j}^{i}}\left(x_{j}\right)}$;

and $\theta=\left[\theta_{1} \theta_{2} \ldots \theta_{N}\right]^{T}$ is fuzzy basis function. 
(b) Step 2: Replace switching functions by the fuzzy functions

After designing the fuzzy system in step 1, the switching functions $u_{s t}^{a}$ and $u_{s t}^{b}$ are replaced by two novel fuzzy replacement functions $\hat{u}_{s t}^{a}$ and $\hat{u}_{s t}^{b}$ designed as

$$
\left\{\begin{array}{l}
\hat{u}_{s t}^{a}=\left|s_{1}\right|^{\frac{1}{2}} \psi_{1}^{T} \theta . \\
\hat{u}_{s t}^{b}=\psi_{2}^{T} \theta t_{c}
\end{array}\right.
$$

Note that $\hat{u}_{s t}^{a}$ and $\hat{u}_{s t}^{b}$ are two fuzzy systems defined by Eq. (20), whose only input is the sliding variable $s_{1}$, and provides the optimal values satisfying $\left|\hat{u}_{s t}^{* a}\right|=\tilde{K}_{1}^{*}|s|^{\frac{1}{2}}$ and $\left|\hat{u}_{s t}^{* b}\right|=\tilde{K}_{2}^{*} t_{c}$ as an output. Here, $\left|\hat{u}_{s t}^{* a}\right|$, and $\left|\hat{u}_{s t}^{* b}\right|$ are the optimal values of $\left|\hat{u}_{s t}^{a}\right|$, and $\left|\hat{u}_{s t}^{b}\right|$, respectively, and $t_{c}$ is the time of state convergence.

(c) Step 3: Design of the adaptive laws

To obtain optimal values $\left|\hat{u}_{s t}^{* a}\right|$ and $\left|\hat{u}_{s t}^{* b}\right|$, proper convergence of the parameters $\psi_{1}$ and $\psi_{2}$ is required. Hence, to govern the convergence of these parameters, the adaptive laws are designed as

$$
\begin{aligned}
& \dot{\psi}_{1}=\beta_{1} s_{1}\left|s_{1}\right|^{\frac{1}{2}} \theta \\
& \dot{\psi}_{2}=\beta_{2} s_{1} \theta t_{c} .
\end{aligned}
$$

Here, $\beta_{1}$ and $\beta_{2}$ are the adaptation parameters that are used to regulate the adaptation speed.

Finally, the auxiliary control input $u(t)$ [Eq. (17)] and the proposed actual control input $\tau$, which is now named as AF-HOSTSMC [Eq. (18)], is represented as

$$
\begin{gathered}
u(t)=-\hat{u}_{s t}^{* a}-\hat{u}_{s t}^{* b}-\frac{2}{3} K_{3} \xi_{2}\left|\xi_{1}\right|^{-1 / 3}, \\
\tau=G_{\text {nom }}^{-1}(t, q)\left\{-H_{n o m}(t, q)-\hat{u}_{s t}^{* a}-\hat{u}_{s t}^{* b}-\frac{2}{3} K_{3} \xi_{2}\left|\xi_{1}\right|^{-1 / 3}\right\} .
\end{gathered}
$$

\subsection{Stability Analysis}

In this subsection, for the closed-loop system Eq. (11), stability proof has been discussed. The proof is divided into two parts: first, it is shown that if the system states deviate from the sliding surface, then $s_{1}=0$ is achieved in finite time, and the sliding mode will be re-established. Second, it is shown that the error during trajectory tracking will reach zero in finite time.

Theorem 4.5: For the system Eq. (11), the proposed control law (AF-HOSTSMC) [Eq. (23)] and adaptive laws [Eq. (21)] will ensure the convergence of the states in finite time, under the presence of uncertainties and disturbances.

\section{Definition 4.6:}

$$
V_{1}=\frac{1}{2} s_{1}^{T} S_{1}+\frac{1}{2 \beta_{1}} \tilde{\psi}_{1}^{T} \tilde{\psi}_{1}+\frac{1}{2 \beta_{2}} \tilde{\psi}_{2}^{T} \tilde{\psi}_{2}
$$

with $\tilde{\psi}_{1,2}=\psi_{1,2}-\psi_{1,2}^{*}$ is an adaption error, where $\psi_{1,2}^{*}$ is the optimal values of $\psi_{1,2}$ and $\beta_{1}, \beta_{2}$ are adaption constants.

The first time-derivative of $V_{1}$ is 


$$
\dot{V}_{1}=s_{1}^{T} \dot{S}_{1}+\frac{1}{\beta_{1}} \tilde{\psi}_{1}^{T} \dot{\psi}_{1}+\frac{1}{\beta_{2}} \tilde{\psi}_{2}^{T} \dot{\psi}_{2}
$$

Using the system Eq. (11) and the control law Eq. (17), the first time-derivative of $s_{1}$ [Eq. (22)] is

$$
\begin{aligned}
\dot{s}_{1} & =\dot{\xi}_{2}+\frac{2}{3} K_{3}\left|\xi_{1}\right|^{-\frac{1}{3}} \dot{\xi}_{1} \\
& =\alpha(t)+\tilde{d}+\frac{2}{3} K_{3}\left|\xi_{1}\right|^{-\frac{1}{3}} \xi_{2} . \\
& =-\hat{u}_{s t}^{a}-\hat{u}_{s t}^{b}+\tilde{d}
\end{aligned}
$$

Rewriting Eq. (26) as

$$
\dot{s}_{1}=-\hat{u}_{s t}^{a}-\hat{u}_{s t}^{* a}+\hat{u}_{s t}^{* a}-\hat{u}_{s t}^{b}-\hat{u}_{s t}^{* b}+\hat{u}_{s t}^{* b}+\tilde{d},
$$

where $\hat{u}_{s t}^{* a}=\left|s_{1}\right|^{\frac{1}{2}} \psi_{1}^{* T} \theta$ and $\hat{u}_{s t}^{* b}=\psi_{2}^{* T} \theta t_{c}$, and then, the equation of $\dot{s}_{1}$ becomes

$$
\dot{s}_{1}=-\left(\psi_{1}-\psi_{1}^{*}\right)^{T}\left|s_{1}\right|^{\frac{1}{2}} \theta-\left(\psi_{2}-\psi_{2}^{*}\right)^{T} \theta t_{c}-\hat{u}_{s t}^{* a}-\hat{u}_{s t}^{* b}+\tilde{d} .
$$

Substituting Eq. (28) into Eq. (25), we get

$$
\dot{V}_{1}=-\tilde{\psi}_{1}^{T} s_{1}\left|s_{1}\right|^{\frac{1}{2}} \theta-\tilde{\psi}_{2}^{T} s_{1} \theta t_{c}-s_{1}\left(\hat{u}_{s t}^{* a}+\hat{u}_{s t}^{* b}\right)+\frac{1}{\beta_{1}} \tilde{\psi}_{1}^{T} \dot{\psi}_{1}+\frac{1}{\beta_{2}} \tilde{\psi}_{2}^{T} \dot{\psi}_{2}+\left|s_{1} \| \tilde{d}\right| .
$$

The above equation can be simplified as

$$
\dot{V}_{1}=-s_{1}\left(\hat{u}_{s t}^{* a}+\hat{u}_{s t}^{* b}\right)+\left|s_{1}\right||\tilde{d}|+\frac{1}{\beta_{1}} \tilde{\psi}_{1}^{T}\left(\dot{\psi}_{1}-\beta_{1} s_{1}\left|s_{1}\right|^{\frac{1}{2}} \theta\right)+\frac{1}{\beta_{2}} \tilde{\psi}_{2}^{T}\left(\dot{\psi}_{2}-\beta_{2} s_{1} \theta t_{c}\right) .
$$

Using Eq. (21), we obtain

$$
\begin{aligned}
\dot{V}_{1} & =-\left|s_{1}\right|\left(\hat{u}_{s t}^{* a}+\hat{u}_{s t}^{* b}\right)+\left|s_{1}\right||\tilde{d}| \\
& =\left|s_{1}\right||\tilde{d}|-\left|s_{1}\right|\left|\left(\tilde{K}_{1}^{*}\left|s_{1}\right|^{\frac{1}{2}}+\tilde{K}_{2}^{*} t_{c}\right)\right| \\
& \leq\left|s_{1}\right||\tilde{d}|-\eta\left|s_{1}\right| \leq 0
\end{aligned}
$$

Thus, it is proved that the proposed AF-HOSTSMC guarantees that $s_{1}(t)=0$ is reached in finite time under the presence of uncertainties and disturbances.

Theorem 4.7: For the system Eq. (11), the proposed control law [Eq. (16)] with sliding manifold [Eq. (15)] ensures that the trajectory tracking error in system states will reach zero in finite time.

Definition 4.8: Using Eqs. (11) and (16), the closed-loop system with states $\xi=\left(\xi_{1}, \xi_{2}, \xi_{3}\right)$ can be represented as

$$
\begin{aligned}
& \dot{\xi}_{1}=\xi_{2} \\
& \dot{\xi}_{2}=-\tilde{K}_{1}\left|s_{1}\right|^{1 / 2} \operatorname{sign}\left(s_{1}\right)+\xi_{3}, \\
& \dot{\xi}_{3}=-\tilde{K}_{2} \operatorname{sign}\left(s_{1}\right)+\rho
\end{aligned}
$$

where perturbation $\rho=\dot{d}$ and $|\rho| \leq \Delta$.

Note that system Eq. (32) is homogeneous of degree $\delta_{v}=-1$ with weights $\alpha=[3,2,1]$. The finite-time convergence proof for the states is initiated by the selection of the following Lyapunov function: 


$$
V_{2}(x)=\gamma_{1}\left|\xi_{1}\right|^{4 / 3}-\gamma_{12}\left\lfloor\xi_{1}\right]^{2 / 3} s_{1}+\gamma_{2}\left|s_{1}\right|^{2}+\gamma_{13}\left\lfloor\xi_{1}\right]^{2 / 3}\left\lfloor\xi_{3}\right]^{2}-\gamma_{23} s_{1}\left\lfloor\xi_{3}\right]^{2}+\gamma_{3}\left|\xi_{3}\right|^{4}
$$

Here, Eq. (33) is homogeneous of degree $\delta_{v}=4$ with weights $\alpha=[3,2,1]$, differentiable almost everywhere except at $\xi_{1}=0$ due to the term $\left\lfloor\left.\xi_{1}\right|^{2 / 3}\right.$ and hence not locally Lipschitz. For convenience, a new vector $A^{T}=\left[\begin{array}{llll}\left\lfloor\xi_{1}\right]^{2 / 3} & s_{1} & \left\lfloor\xi_{3}\right. & \rceil^{2}\end{array}\right]$ is introduced to express Eq. (33) in a quadratic form, i.e. $V_{2}(x)=A^{T} \Gamma A$, [28] where

$$
\Gamma=\left[\begin{array}{ccc}
\gamma_{1} & -\frac{1}{2} \gamma_{12} & \frac{1}{2} \gamma_{13} \\
-\frac{1}{2} \gamma_{12} & \gamma_{2} & -\frac{1}{2} \gamma_{23} \\
\frac{1}{2} \gamma_{13} & -\frac{1}{2} \gamma_{23} & \gamma_{3}
\end{array}\right]
$$

This clearly shows that $V_{2}(x)$ is positive definite and radially unbounded iff $\Gamma>0$. This condition will be satisfied only if the following relations hold:

$$
\left\{\begin{array}{l}
\gamma_{1}>0, \quad \gamma_{1} \gamma_{2}-\frac{1}{4} \gamma_{12}^{2}>0, \\
\gamma_{1}\left(\gamma_{2} \gamma_{3}-\frac{1}{4} \gamma_{23}^{2}\right)+\frac{1}{2} \gamma_{12}\left(-\frac{\gamma_{12}}{2} \gamma_{3}+\frac{1}{4} \gamma_{13} \gamma_{23}\right)+\frac{1}{2} \gamma_{13}\left(\frac{1}{4} \gamma_{12} \gamma_{23}-\frac{1}{2} \gamma_{2} \gamma_{13}\right)>0 .
\end{array}\right.
$$

The derivative of Lyapunov function [Eq. (33)], along the trajectories of the perturbed system Eq. (32), is

$$
\begin{aligned}
\dot{V}_{0}(\xi) & \left.\left.=\beta_{1} L \xi_{1}\right\rceil^{\frac{1}{3}} \xi_{2}-\beta_{2}\left|\xi_{1}\right|^{-\frac{1}{3}} \xi_{2}^{2}-2 K_{1} \gamma_{2}\left|s_{1}\right|^{\frac{3}{2}}-\gamma_{23}\left|\xi_{3}\right|^{3}-\beta_{3}\left|\xi_{1}\right|^{-\frac{1}{3}} \xi_{2} L \xi_{3}\right\rceil^{2} \\
& \left.\left.+K_{1} \gamma_{12} L \xi_{1}\right\rceil^{\frac{2}{3}}\left\lfloor s_{1}\right\rceil^{\frac{1}{2}}-\tilde{\beta}_{4} L \xi_{1}\right\rceil^{\frac{2}{3}} \xi_{3}+\tilde{\beta}_{5} \xi_{3} s_{1}+\gamma_{23} K_{1}\left\lfloor\left. s_{1}\right|^{\frac{1}{2}}\left\lfloor\xi_{3}\right\rceil^{2}-\tilde{\beta}_{6} L \xi_{3}\right\rceil^{3}\left\lfloor s_{1}\right\rceil^{0},
\end{aligned}
$$

where

$$
\left\{\begin{array}{l}
\beta_{1}=\frac{4}{3} \gamma_{1}-\frac{4}{3} K_{3} \gamma_{12}+\frac{4}{3} \gamma_{2} K_{3}^{2} \\
\beta_{2}=\frac{2}{3} \gamma_{12}-\frac{4}{3} K_{3} \gamma_{2} \\
\beta_{3}=\frac{2}{3} \gamma_{23} K_{3}-\frac{2}{3} \gamma_{13} \\
\tilde{\beta}_{4}=\gamma_{12}+2 \gamma_{13} K_{2}\left\lfloor s_{1}\right\rceil^{0}\left\lfloor x_{3}\right\rceil^{0}-2 \gamma_{13}\left\lfloor x_{3}\right\rceil^{0} \rho \\
\tilde{\beta}_{5}=2 \gamma_{2}+2 \gamma_{23} K_{2}\left\lfloor s_{1}\right\rceil^{0}\left\lfloor x_{3}\right\rceil^{0}-2 \gamma_{23}\left\lfloor x_{3}\right\rceil^{0} \rho \\
\tilde{\beta}_{6}=4 K_{2} \gamma_{3}-4 \gamma_{3} \rho\left\lfloor s_{1}\right\rceil^{0}
\end{array} .\right.
$$

Note that $\dot{V}_{0}(\xi)$ is a discontinuous function having degree of homogeneity $\delta_{v}=3$ with weights $\alpha=[3,2,1]$. Moreover, in Eq. (35), controller gains $\tilde{K}_{1}$ and $\tilde{K}_{2}$ are assumed (implicitly) constant and hence denoted as $K_{1}$ and $K_{2}$, respectively, for analysis.

In the work of Moreno [28], it has been shown that by solving few non-linear inequalities, the values of the gains $\left(K_{1}, K_{2}, K_{3}\right)$ and the Lyapunov function parameters $\left(\gamma_{1}, \gamma_{2}, \gamma_{3}, \gamma_{12}, \gamma_{23}, \gamma_{13}\right)$ can be obtained, which will make the origin $\xi=0$ finite time stable in spite of bounded perturbation $|\rho| \leq \Delta$. Moreover, from Ref. [14, Theorem 1, p. 24] it follows that, with the help of graphical interpretation method, the non-unique values of above-mentioned gains and Lyapunov function parameters can be obtained, which will adjust the parameters $\left(\beta_{i}, i=1,2, \ldots 6\right)$ of Eq. (36) such that Eq. (35) becomes negative definite, i.e. $\dot{V}_{0}(\xi)<0$ for every value of the perturbation $|\rho| \leq \Delta$. In view of this, the function $\Omega(\xi)$ 


$$
\Omega(\xi)=\frac{-\dot{V}_{0}(\xi)}{V_{0}^{\frac{3}{4}}(\xi)}
$$

has a homogeneity degree $\delta_{\Omega} \overline{\overline{2}} 0$, and therefore, all values of the function are available on the unity homogeneous ball $B_{h}=\left\{\left.\xi \in R^{3}|| \xi_{1}\right|^{\overline{3}}+\left|\xi_{2}\right|+\left|\xi_{3}\right|^{2}=1\right\}$. On $B_{h}$, the function $V_{0}(\xi)$ is continuous, while the function $-\dot{V}_{0}(\xi)$ is continuous almost everywhere except at $\xi_{1}=0$ where it is $+\infty$. Additionally, both of them have different values other than zero on ball $B_{h}$. Therefore, $\Omega(\xi)$ has a positive minimum such that

$$
\varpi=\min _{\xi \in B_{h}} \Omega(\xi) .
$$

To this end, it implies that

$$
\frac{-\dot{V}_{0}(\xi)}{V_{0}^{\frac{3}{4}}(\xi)} \geq \varpi \text { or } \quad \dot{V}_{0}(\xi) \leq-\varpi V_{0}^{3 / 4}(\xi) \text {. }
$$

Hence, the function $\dot{V}_{0}(\xi)$ satisfies the differential inequality Eq. (38) for some positive $\varpi$, and it is a Lyapunov function for the system Eq. (11), whose trajectories, starting from any initial condition $\left(\xi_{0}\right)$, converge to the origin in the presence of disturbances in finite time $T$, calculated as [28]

$$
T\left(\xi_{0}\right) \leq \frac{4}{\varpi} V^{\frac{1}{4}}\left(\xi_{0}\right)
$$

Hence, in light of Definition 3.2, it is proved that trajectories of the system Eq. (11), starting from any initial condition, converges to the origin, in the presence of disturbances in finite time with the help of the proposed controller [Eq. (16)] and adaptive laws [Eq. (21)]. The overall control strategy for trajectory tracking control with the help of proposed AF-HOSTSMC is shown in Figure 1.

\section{Results and Discussion}

In this section, the efficacy of the proposed AF-HOSTSMC is analyzed on a two-link planar RM (MIMO) system shown in Figure 2. The simulations results are obtained in the MATLAB-Simulink environment using ODE 4 solver with a fixed step size of $0.005 \mathrm{~s}$. Table 1 lists the physical attributes of the manipulator used for the simulation analysis. The dynamic equation of the two-link manipulator system with uncertainty is represented as [40]

$$
M(q) \ddot{q}+C(q, \dot{q}) \dot{q}+G(q)+F(q, \dot{q})=\tau+\tau_{d},
$$

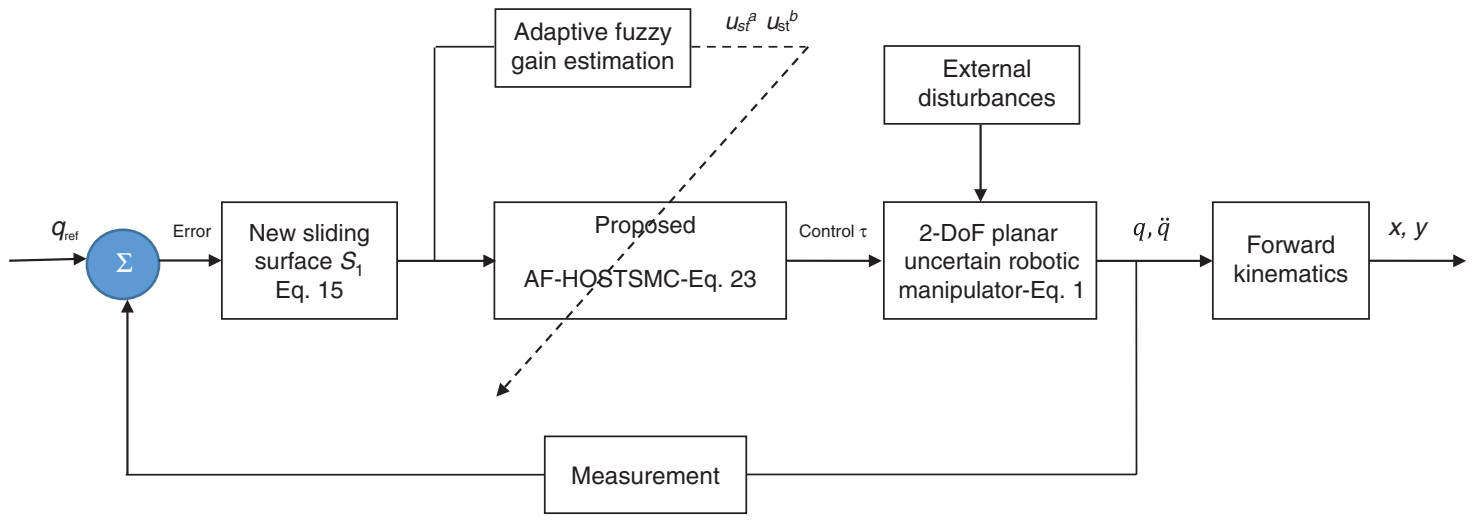

Figure 1: Block Diagram of the Proposed Control Strategy. 


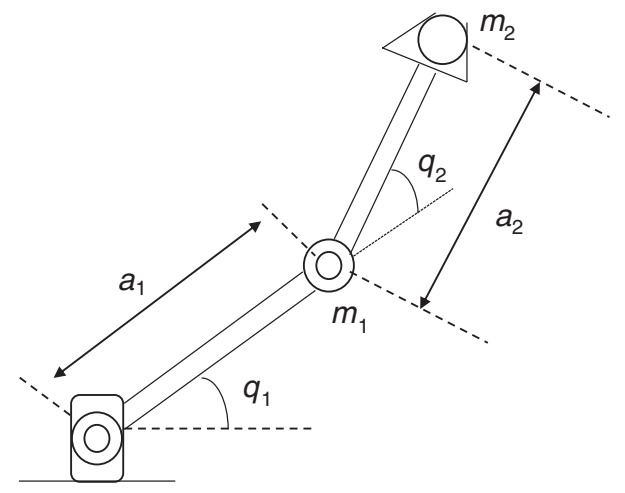

Figure 2: Configuration of the Two-Link Robotic Manipulator.

Table 1: Parameters of 2-DoF Robotic Manipulator.

\begin{tabular}{lll}
\hline Symbol & Definition & Value \\
\hline$m_{1}$ & Nominal mass of link 1 & $1 \mathrm{~kg}$ \\
$m_{2}$ & Nominal mass of link 2 & $1.5 \mathrm{~kg}$ \\
$l_{1}$ & Length of link 1 & $1 \mathrm{~m}$ \\
$l_{2}$ & Length of link 2 & $0.8 \mathrm{~m}$ \\
$l_{1}$ & Moment of inertia (y-axis) & $12 \mathrm{~kg} \cdot \mathrm{m}^{2}$ \\
$l_{2}$ & Moment of inertia (z-axis) & $26 \mathrm{~kg} \cdot \mathrm{m}^{2}$ \\
$g$ & Gravitational constant & $9.8 \mathrm{~m} / \mathrm{s}^{2}$ \\
\hline
\end{tabular}

where

$$
M(q)=\left[\begin{array}{ll}
M_{11}(q) & M_{12}(q) \\
M_{21}(q) & M_{22}(q)
\end{array}\right] ; C(q, \dot{q}) \dot{q}=\left[\begin{array}{c}
C_{11}(q) \\
C_{21}(q)
\end{array}\right] ; G(q)=\left[\begin{array}{c}
G_{11}(q) \\
G_{21}(q)
\end{array}\right],
$$

where

$$
\begin{aligned}
& M_{11}(q)=m_{2} a_{1}^{2}+2 m_{2} a_{1} a_{2} \cos \left(q_{2}\right)+\left(m_{1}+m_{2}\right) a_{1}^{2}+I_{1} \\
& M_{12}(q)=M_{21}(q)=m_{2} a_{2}^{2}+m_{2} a_{1} a_{2} \cos \left(q_{2}\right) ; M_{22}(q)=m_{2} a_{2}^{2}+I_{2} \\
& C_{11}(q)=-m_{2} a_{1} a_{2} \sin \left(q_{2}\right) \dot{q}_{2}^{2}-2 m_{2} a_{1} a_{2} \sin \left(q_{2}\right) \dot{q}_{1} \dot{q}_{2} \\
& C_{21}(q)=m_{2} a_{1} a_{2} \sin \left(q_{2}\right) \dot{q}_{2}^{2} \\
& G_{11}(q)=m_{2} a_{2} g \cos \left(q_{1}+q_{2}\right)+\left(m_{1}+m_{2}\right) a_{1} g \cos \left(q_{1}\right) \\
& G_{21}(q)=m_{2} a_{2} g \cos \left(q_{1}+q_{2}\right) .
\end{aligned}
$$

Here, $q(t)=\left[q_{1}(t), q_{2}(t)\right]^{T}$ is the position vector, $M(q)$ is the inertia matrix, $C(q, \dot{q})$ is the centripetal Coriolis matrix, $G(q)$ is the gravity vector, and $\tau=\left[\tau_{1}, \tau_{2}\right]^{T}$ is the applied torque vector. Furthermore, the manipulator has four states $x_{1}(t)=q_{1}(t), x_{2}(t)=\dot{q}_{1}(t), x_{3}(t)=q_{2}(t), x_{4}(t)=\dot{q}_{2}(t)$, two output states $y_{1}(t)=q_{1}(t), y_{2}(t)=q_{2}(t)$, and two inputs $u_{1}(t)=\tau_{1}, u_{2}(t)=\tau_{2}$. The frictional effects in joints, including the viscous friction and the Coulomb friction torques, are selected as $F(q, \dot{q})=\left[8 \sin \left(\dot{q}_{1}\right)+1.3 \operatorname{sign}\left(\dot{q}_{1}\right) \quad 8 \sin \left(\dot{q}_{2}\right)+1.3 \operatorname{sign}\left(\dot{q}_{2}\right)\right]^{T}$. The external disturbances are selected as $\tau_{d}=[0.5 \sin (2 t) 0.8 \cos (2 t)]^{T}$.

The reference signals are chosen as $\left[q_{1 d} q_{d 2}\right]^{T}=[0.3 \sin (t) 0.5 \sin (t)]^{T}$. For simulation, the initial states of RM are selected as $q_{1}(0)=0.1, q_{2}(0)=0.1, \dot{q}_{1}(0)=0, \dot{q}_{2}(0)=0$. The design parameters of the proposed AF-HOSTSMC are tabulated in Table 2. The membership function for fuzzy systems is chosen as 


$$
\mu_{A_{j}^{i}}\left(x_{j}\right)=\exp \left(-\left(\frac{x_{i}-\bar{x}_{i}^{l}}{\pi / 24}\right)^{2}\right),
$$

where $\bar{x}_{i}^{l}$ are $(-\pi / 6),(-\pi / 12), 0,(\pi / 12)$, and $(\pi / 6)$, respectively; $i=1,2, \ldots, 5 ; A_{i}^{l}$ is the fuzzy set including NB, NS, ZO, PS, PB, which belong to $l^{\text {th }}$ fuzzy rule as shown in Figure 3.

Simulation results obtained by applying the proposed controller [Eq. (23)] are shown in Figures 4-7. From Figure 4, it is observed that both joints 1 and 2 track the reference trajectory faithfully. The control inputs

Table 2: Proposed Controller (AF-HOSTSMC) Parameters.

\begin{tabular}{ll}
\hline Controller & Tuning parameter \\
\hline Proposed controller [Eq. (23)] & $\beta_{1}=\operatorname{diag}(90,90), \beta_{2}=\operatorname{diag}(45,45)$ \\
& $\tilde{K}_{1}^{*}=\operatorname{diag}(250,250), \tilde{K}_{2}^{*}=\operatorname{diag}(255,255)$, \\
& $K_{3}=\operatorname{diag}(10,10)$ \\
\hline
\end{tabular}

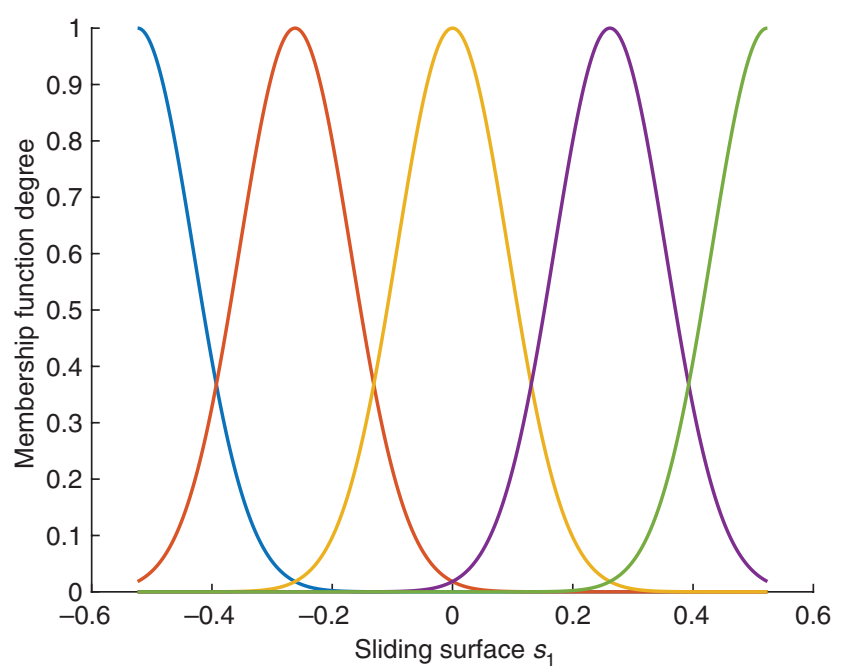

Figure 3: Membership Function.
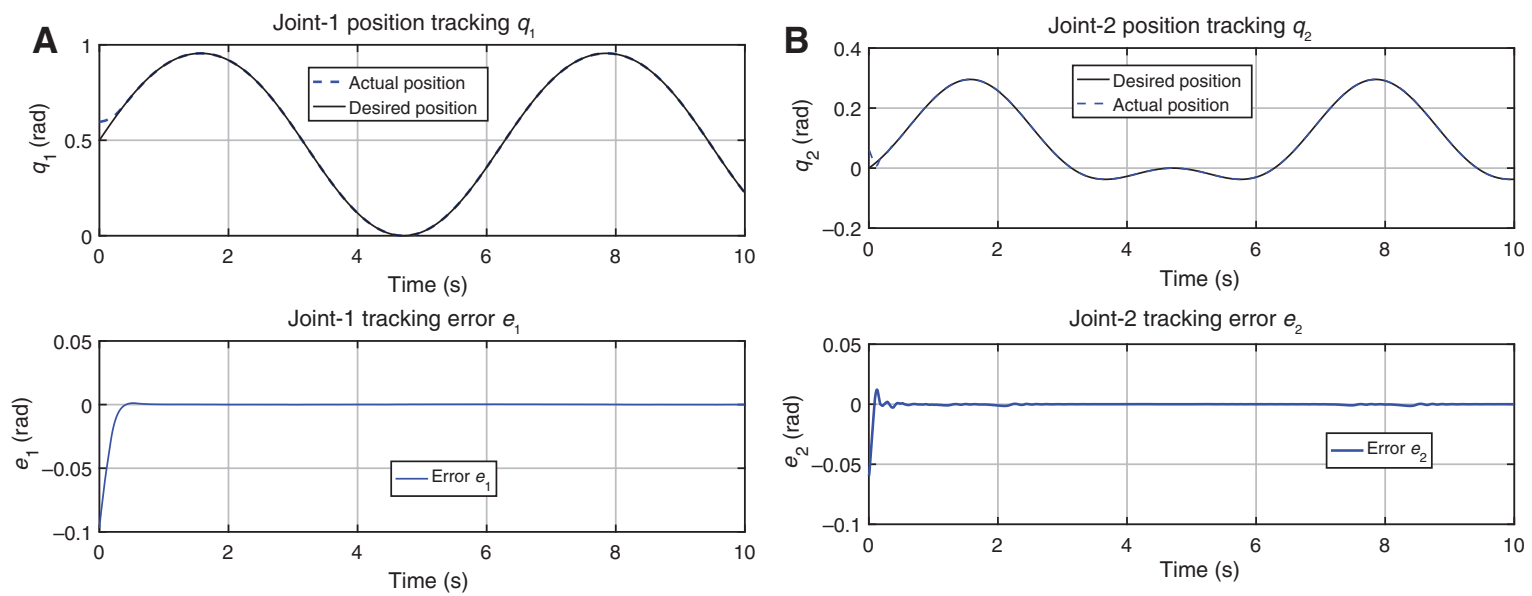

Figure 4: Closed-Loop System Trajectories Using the Proposed AF-HOSTSMC [Eq. (23)].

(A) Performance of state $q_{1}$ and its error $e_{1}$. (B) Performance of state $q_{2}$ and its error $e_{2}$. 

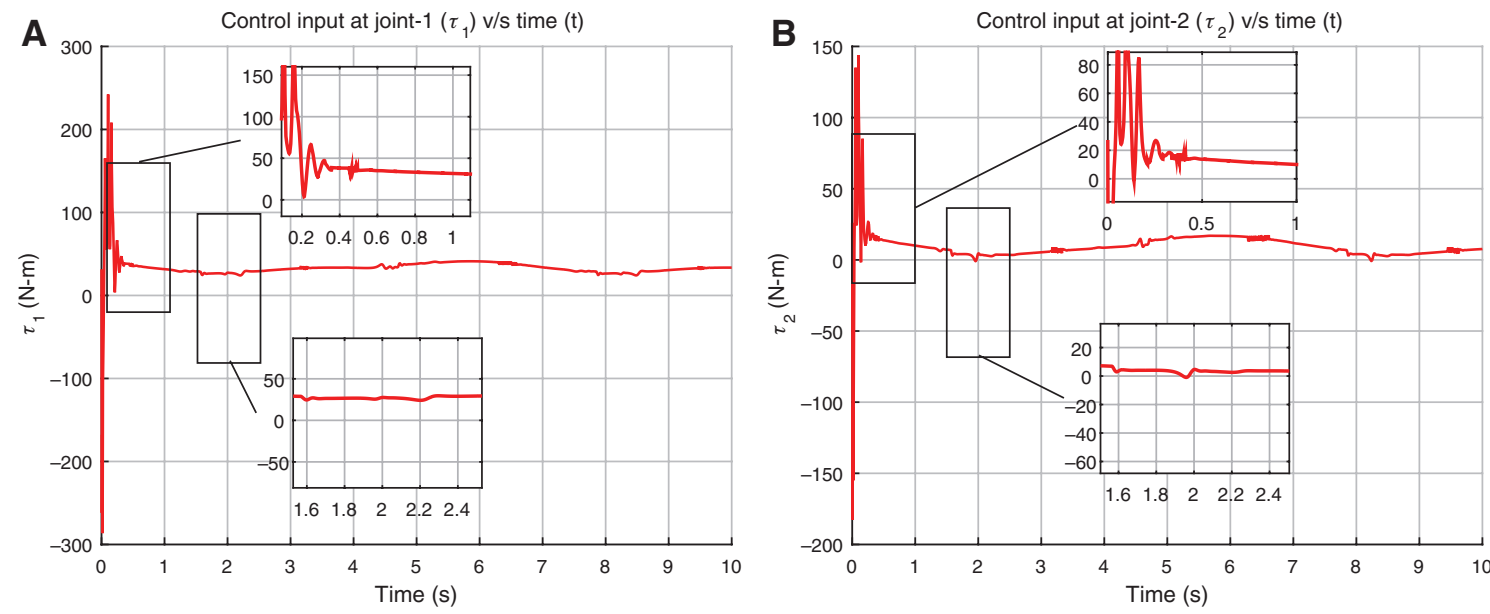

Figure 5: Trajectory of the Control Input Using the Proposed AF-HOSTSMC [Eq. (23)].

(A) Control input $\tau_{1}$ vs. time at joint 1. (B) Control input $\tau_{2}$ vs. time at joint 2 .
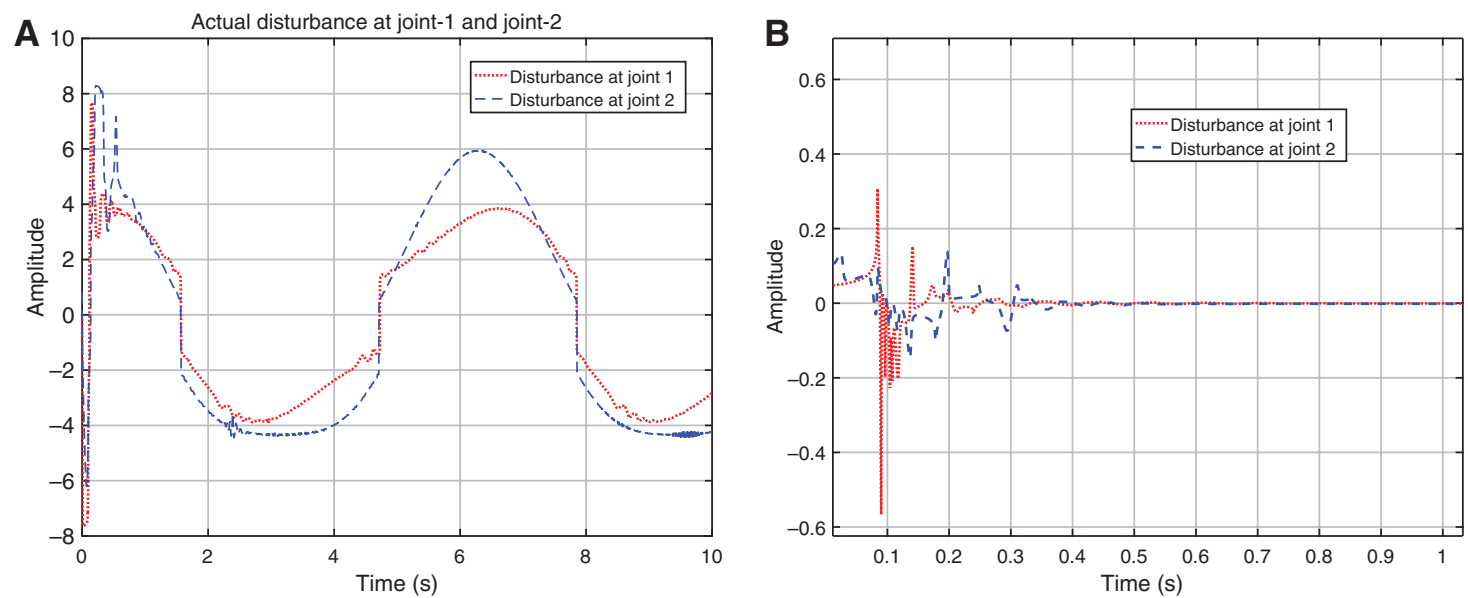

Figure 6: Profile of Actual Disturbance and Estimated Disturbance at Joint 1 and Joint 2.

(A) Actual disturbances present in the system. (B) Estimated and cancelled disturbances by the proposed controller.
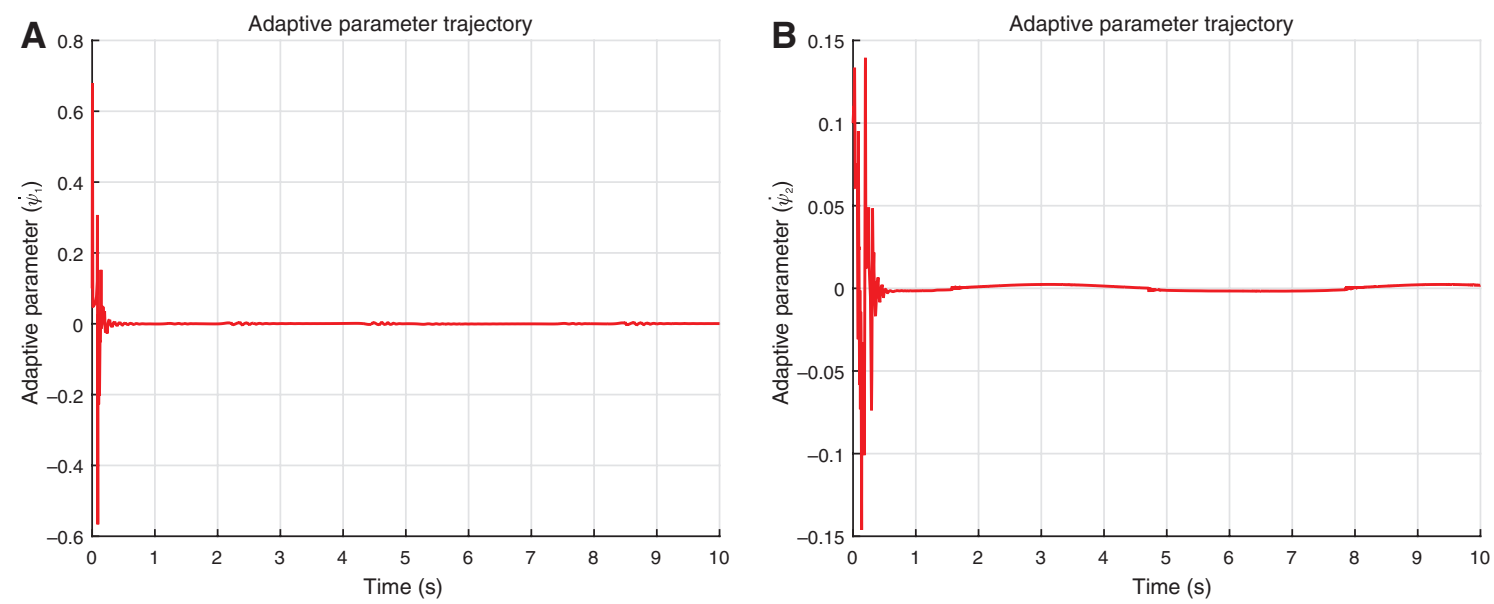

Figure 7: Time History of the Adaptive Parameters [Eq. (21)]. (A) Adaptive parameter $\dot{\psi}_{1}$. (B) Adaptive parameter $\dot{\psi}_{2}$. 
applied to both joints is continuous input and with initial short span of chattering, i.e. approximately $0.4 \mathrm{~s}$ and after that the chattering phenomenon is substantially eliminated in both joints as evident in Figure 5. Figure $6 \mathrm{~A}$ and $\mathrm{B}$ represent the actual disturbances present in the joints and the effectiveness of the proposed controller in canceling these external perturbations, respectively. Clearly, the proposed controller is capable of rejecting the disturbances present in the system, resulting in the very quick and finite position error convergence to zero. Furthermore, due to the presence of frictional uncertainties and external disturbances, the states are likely to deviate from the chosen sliding surface, and therefore, the convergence plots of $\dot{\psi}_{1}$ and $\dot{\psi}_{2}$ in Figure 7A and B show that adaptive parameters are continuously updating the fuzzy system till the sliding mode is attained.
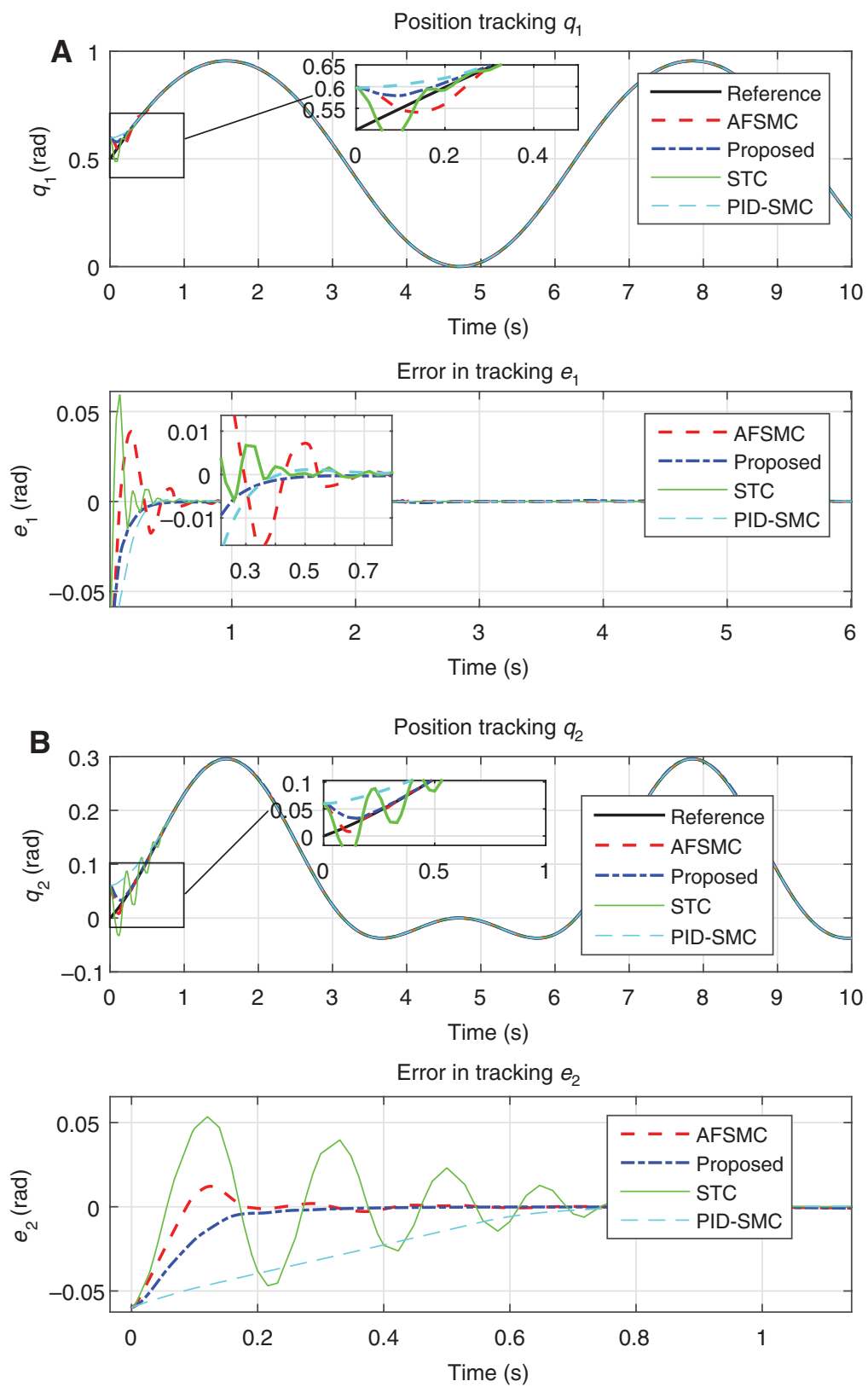

Figure 8: Closed-Loop System Trajectories Using the Proposed AF-HOSTSMC [Eq. (23)], AFSMC [40], STC [Eq. (13)], and PID-SMC [18].

(A) Performance of state $q_{1}$ and its error $e_{1}$. (B) Performance of state $q_{2}$ and its error $e_{2}$ 
To illustrate the efficacy of the proposed controller, a comparison has been performed with the following existing sliding mode controllers applied to the same RM system:

- [40] An AFSMC, having a fuzzy logic estimator to approximate lumped uncertainty in equivalent control, with control law $\tau=f+\hat{F}+\lambda_{0} s+\lambda_{1} \operatorname{sign}(s)$ with sliding surface $s=\dot{\sigma}+\beta_{1} \sigma+\beta_{2} \int_{0}^{t} \sigma d t, \sigma$ is an error vector [Eq. (7)], $f$ is lumped uncertainty, $\hat{F}$ is output from fuzzy logic estimator, and $\beta_{1}=\operatorname{diag}(10,10), \beta_{2}=\operatorname{diag}(0.1$, $0.1), \lambda_{0}=\operatorname{diag}(10,10)$, and $\lambda_{1}=\operatorname{diag}(1,1.5)$.

- A conventional super-twisting SMC (STC) with control law [Eq. (13)], sliding surface $s=\dot{\sigma}+\beta_{1} \sigma$, where $\sigma$ is an error vector [Eq. (7)], $\gamma=0.5, \beta_{1}=\operatorname{diag}(10,10), \lambda=\operatorname{diag}(10,10)$, and $\alpha=\operatorname{diag}(10,15)$.

- [18] A conventional PID-SMC with control law $\tau=f+\lambda_{0} s+\lambda_{1} \operatorname{sign}(s)$, sliding surface $s=\dot{\sigma}+\beta_{1} \sigma+\beta_{2} \int_{0}^{t} \sigma d t$, where $\sigma$ is an error vector [Eq. (7)], $f$ is lumped uncertainty, $\beta_{1}=\operatorname{diag}(10,10), \beta_{2}=\operatorname{diag}(0.1,0.1), \lambda_{0}=\operatorname{diag}$ $(10,10)$, and $\lambda_{1}=\operatorname{diag}(10,15)$.
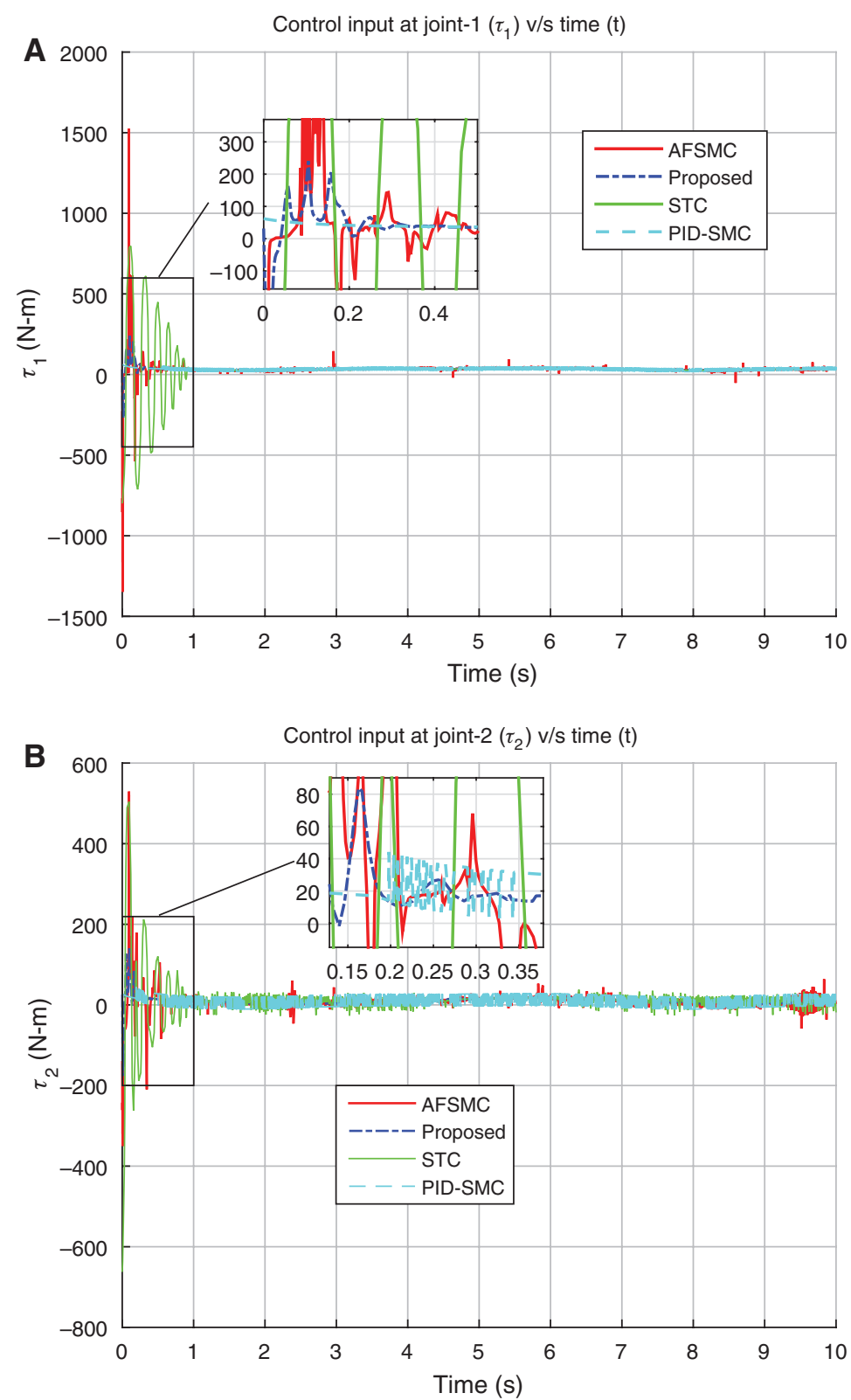

Figure 9: Trajectory of the Control Input Using the Proposed AF-HOSTSMC [Eq. (23)], AFSMC [40], STC [Eq. (13)], and PID-SMC [18]. (A) Control input $\tau_{1}$ vs. time at joint 1 . (B) Control input $\tau_{2}$ vs. time at joint 2. 
With the same initial conditions, the trajectory tracking response and the control input profile, obtained by using the above-mentioned controllers, are illustrated in Figures 8 and 9, respectively. Figure 8A and $\mathrm{B}$ show the comparison of the time history of the states and their errors, obtained from the application of the proposed AF-HOSTSMC and rest of the controllers (AFSMC, STC, and PID-SMC). Clearly, by using the proposed controller, the states $\left|q_{1}\right| \leq 0.149 \times 10^{-4}$ and $\left|q_{2}\right| \leq 0.286 \times 10^{-4}$ are reached in the finite times of $t=0.3895 \mathrm{~s}$ and $0.491 \mathrm{~s}$, respectively, which were faster than the remaining controllers. Moreover, from Figure 9A and B, it is observed that the major drawback of the other controllers (AFSMC, STC, and PID$\mathrm{SMC}$ ) is the high-frequency chattering present in the control input. The variation in the control input, which is quite high in other controllers, is undesirable as it can damage the actuator or it may get saturated. On contrary, the proposed controller input is smooth and chattering free. Moreover, another design constraint the other controllers suffer from is that the uncertainty bounds need to be known a priori. Moreover, AFSMC and STC show larger overshoot than the proposed AF-HOSTSMC, which is not desirable for high-precision tracking. Table 3 summarizes the performance of the all four controllers for both joint 1 and joint 2, from which is evident that the proposed AF-HOSTSMC method exhibits better control performance, e.g. no overshoot, faster state convergence, and negligible and bounded chattering than other controllers.

Further, to have a more detailed comparison between the performance of the proposed controller and that of other controllers, an analysis has been performed for input and output characteristics in terms of standard performance indices.

To evaluate the output performance, the performance index selected is integrated absolute error (IAE) of the output, which is given as IAE $=$ sum of the absolute error values $=\sum_{k=1}^{n}\left\|q(k)-q_{d}(k)\right\|$, where $n$ is the number of sampling instants and $q_{d}$ is the desired positions of the respective joints at the $k^{\text {th }}$ sampling instant.

For computing input usage, the total variation (TV) [33] of the input $u(t)$ is calculated as

$$
\mathrm{TV}=\sum_{i=1}^{n}\left\|u_{i+1}-u_{i}\right\|
$$

which is desired to have a small value. The total variation measures the smoothness of a signal. A large value of TV represents more excessive input utilization or, in other words, a more complicated controller. Moreover, the energy of the input signal is evaluated by using the two-norm method ( $L_{2}$ norm). The control energy is expected to be as small as possible.

The output and input performances are calculated for the period from 0 to $10 \mathrm{~s}$ with a sampling time of $0.05 \mathrm{~s}$. The results obtained from the proposed AF-HOSTSM controller along with other controllers for the two-link RM are summarized in Table 4. It is noted that the proposed controller offers comparable tracking performance by applying a smoother control input having minimal total variation as compared to the other sliding mode controllers.

Hence, by comparison, it is revealed that the proposed controller supports our important claim of having high-performance criterion and chattering-less control even in the presence of various uncertainties and external disturbances.

Table 3: Controller Performance Summary.

\begin{tabular}{|c|c|c|c|c|c|c|}
\hline \multirow[t]{2}{*}{ Controllers } & \multicolumn{2}{|c|}{ Steady precision } & \multicolumn{2}{|c|}{ Convergence time } & \multicolumn{2}{|c|}{ Max. overshoot } \\
\hline & $\begin{array}{r}\text { Joint } 1 \\
\left(\times 10^{-4}\right)\end{array}$ & $\begin{array}{r}\text { Joint } 2 \\
\left(\times 10^{-4}\right)\end{array}$ & $\begin{array}{r}\text { Joint } 1 \\
\text { (s) }\end{array}$ & $\begin{array}{r}\text { Joint } 2 \\
(\mathrm{~s})\end{array}$ & $\begin{array}{r}\text { Joint } 1 \\
\text { (rad) }\end{array}$ & $\begin{array}{r}\text { Joint } 2 \\
\text { (rad) }\end{array}$ \\
\hline Proposed [Eq. (23)] & 0.149 & 0.286 & 0.3895 & 0.491 & - & - \\
\hline AFSMC [40] & 1.958 & 1.111 & 0.6968 & 0.681 & 0.03873 & 0.1209 \\
\hline STC [Eq. (13)] & 2.058 & 1.359 & 0.7719 & 1.066 & 0.05941 & 0.0535 \\
\hline PID-SMC [18] & 1.997 & 1.798 & 0.4221 & 0.863 & - & - \\
\hline
\end{tabular}


Table 4: Comparison of the Controller Input-Output Performances for the System [Eq. (40)].

\begin{tabular}{|c|c|c|c|c|c|c|}
\hline \multirow[t]{2}{*}{ Controllers } & \multicolumn{2}{|c|}{ IAE $\left(\times 10^{-3}\right)$} & \multicolumn{2}{|r|}{ TV } & \multicolumn{2}{|c|}{$L_{2}$ norm of input } \\
\hline & Joint 1 & Joint 2 & Joint 1 & Joint 2 & Joint 1 & Joint 2 \\
\hline Proposed [Eq. (23)] & 6.451 & 0.7393 & 1.988 & 0.4436 & 45.05 & 11.08 \\
\hline AFSMC [40] & 9.312 & 2.5015 & 6.191 & 6.9422 & 45.76 & 11.56 \\
\hline STC [Eq. (13)] & 20.17 & 5.1278 & 34.725 & 11.06 & 83.24 & 49.87 \\
\hline PID-SMC [18] & 16.36 & 18.08 & 2.139 & 1.456 & 93.47 & 30.95 \\
\hline
\end{tabular}

\section{Conclusions}

A novel robust adaptive fuzzy high-order super-twisting sliding mode controller for the RM (MIMO) system with frictional uncertainty, other uncertainties, and external disturbances is proposed in this paper. The new sliding manifold based on a homogeneous concept is designed. An adaptive fuzzy mechanism, to adjust the controller gain, is designed, which provides two fuzzy functions that substitute switching parameters of modified super-twisting SMC. The combined controller provides third-order features with only position and velocity state measurement. The proposed controller provides better disturbance rejection, generates continuous control, has no overshoot, and has finite-time convergence. Prior knowledge of disturbances is not essential except the requirement for the Lipschitz condition. The simulation results show that the proposed controller successfully satisfies the performance requirement for precise RM trajectory tracking. The proposed controller is compared against recently developed SMC-based controllers. Finally, the study proves the suitability of novel scheme in application to other higher complex non-linear uncertain mechanical systems.

\section{Bibliography}

[1] F. Amato, M. Ariola and P. Dorato, Finite-time control of linear systems subject to parametric uncertainties and disturbances, Automatica 37 (2001), 1459-1463.

[2] G. Bartolini, A. Ferrara, A. Levant and E. Usai, On second order sliding mode controllers, in: Variable Structure Systems, Sliding Mode and Nonlinear Control, pp. 329-350, Springer, Berlin, 1999.

[3] M. Benbrahim, N. Essounbouli, A. Hamzaoui and A. Betta, Adaptive type-2 fuzzy sliding mode controller for SISO nonlinear systems subject to actuator faults, Int. J. Autom. Comput. 10 (2013), 335-342.

[4] S. P. Bhat and D. S. Bernstein, Finite-time stability of homogeneous systems, in: Proceedings of the American Control Conference, 4, pp. 2513-2514, American Automatic Control Council, 1997.

[5] L. M. Capisani, A. Ferrara and L. Magnani, Second order sliding mode motion control of rigid robot manipulators, in: 46th IEEE Conference on Decision and Control, 2007, pp. 3691-3696, IEEE, 2007.

[6] A. Cavallo and C. Natale, High-order sliding control of mechanical systems: theory and experiments, Control Eng. Pract. 12 (2004), 1139-1149.

[7] M. Corless, Control of uncertain nonlinear systems, J. Dynam. Syst. Meas. Control 115 (1993), 362-372.

[8] J. J. Craig, Introduction to Robotics: Mechanics and Control, 3, Pearson Prentice Hall, Upper Saddle River, NJ, 2005.

[9] N. Derbel and A. M. Alimi, Design of a sliding mode controller by fuzzy logic, Int. J. Robot. Autom. 21 (2006), 241-246.

[10] Z. Doulgeri, Sliding regime of a nonlinear robust controller for robot manipulators, IEE Proc. Control Theory Appl. 146 (1999), 493-498.

[11] K. S. Eom, I. H. Suh, W. K. Chung and S. R. Oh, Disturbance observer based force control of robot manipulator without force sensor, in: IEEE International Conference on Robotics and Automation, 1998. Proceedings. 1998, 4, pp. 3012-3017, IEEE, 1998.

[12] M. Ertugrul and O. Kaynak, Neuro sliding mode control of robotic manipulators, Mechatronics 10 (2000), 239-263.

[13] L. M. Fridman, Singularly perturbed analysis of chattering in relay control systems, IEEE Trans. Autom. Control 47 (2002), 2079-2084.

[14] L. Fridman, J. A. Moreno, B. Bandyopadhyay, S. Kamal and A. Chalanga, Continuous Nested Algorithms: The Fifth Generation of Sliding Mode Controllers, pp. 5-35, Springer International Publishing, Cham, 2015.

[15] M. Galicki, Finite-time control of robotic manipulators, Automatica 51 (2015), 49-54.

[16] Y. Hong, J. Huang and Y. Xu, On an output feedback finite-time stabilization problem, IEEE Trans. Autom. Control 46 (2001), 305-309. 
[17] P. Husek, Adaptive fuzzy sliding mode control for uncertain nonlinear systems, in: Advances in Control and Optimization of Dynamical Systems, 3, pp. 540-545, 2014.

[18] E. M. Jafarov, M. N. A. Parlakçi and Y. Istefanopulos, A new variable structure PID-controller design for robot manipulators, IEEE Trans. Control Syst. Technol. 13 (2005), 122-130.

[19] H. Javaheri and G. R. Vossoughi, Sliding mode control with online fuzzy tuning: application to a robot manipulator, in: Mechatronics and Automation, 2005 IEEE International Conference, 3, pp. 1357-1362, IEEE, 2005.

[20] D. Katic and M. Vukobratovic, Intelligent Control of Robotic Systems, 25, Springer Science \& Business Media, 2013.

[21] O. Kaynak, K. Erbatur and M. Ertugnrl, The fusion of computationally intelligent methodologies and sliding-mode control a survey, IEEE Trans. Ind. Electron. 48 (2001), 4-17.

[22] M. H. Khooban and M. R. Soltanpour, Swarm optimization tuned fuzzy sliding mode control design for a class of nonlinear systems in presence of uncertainties, J. Intell. Fuzzy Syst. 24 (2013), 383-394.

[23] A. Levant, Sliding order and sliding accuracy in sliding mode control, Int. J. Control 58 (1993), 1247-1263.

[24] A. Levant, Higher-order sliding modes, differentiation and output-feedback control, Int. J. Control 76 (2003), 924-941.

[25] T. H. S. Li and Y. C. Huang, MIMO adaptive fuzzy terminal sliding-mode controller for robotic manipulators, Inform. Sci. 180 (2010), 4641-4660.

[26] M. Liu, Decentralized control of robot manipulators: nonlinear and adaptive approaches, IEEE Trans. Autom. Control 44 (1999), 357-363.

[27] M. Manceur, N. Essounbouli and A. Hamzaoui, Second-order sliding fuzzy interval type-2 control for an uncertain system with real application, IEEE Trans. Fuzzy Syst. 20 (2012), 262-275.

[28] J. A. Moreno, Lyapunov function for Levant's second order differentiator, in: CDC, pp. 6448-6453, 2012.

[29] R. Ortega and M. W. Spong, Adaptive motion control of rigid robots: a tutorial, Automatica 25 (1989), 877-888.

[30] A. Sabanovic, Variable structure systems with sliding modes in motion control - a survey, IEEE Trans. Ind. Inform. 2 (2011), 212-223.

[31] V. Santibañez, R. Kelly and M. A. Llama, A novel global asymptotic stable set-point fuzzy controller with bounded torques for robot manipulators, IEEE Trans. Fuzzy Syst. 13 (2005), 362-372.

[32] S. E. Shafiei and S. Sepasi, Incorporating sliding mode and fuzzy controller with bounded torques for set-point tracking of robot manipulators, Elektron. Elektrotech. 104 (2015), 3-8.

[33] S. Skogestad, Simple analytic rules for model reduction and PID controller tuning, J. Process Control 13 (2003), 291-309.

[34] J. J. E. Slotine, The robust control of robot manipulators, Int. J. Robot. Res. 4 (1985), 49-64.

[35] J. J. E. Slotine and W. Li, On the adaptive control of robot manipulators, Int. J. Robot. Res. 6 (1987), 49-59.

[36] M. R. Soltanpour and M. H. Khooban, A particle swarm optimization approach for fuzzy sliding mode control for tracking the robot manipulator, Nonlin. Dynam. 74 (2013), 467-478.

[37] M. R. Soltanpour, M. H. Khooban and M. R. Khalghani, An optimal and intelligent control strategy for a class of nonlinear systems: adaptive fuzzy sliding mode, J. Vibr. Control 22 (2016), 159-175.

[38] M. R. Soltanpour, B. Zolfaghari, M. Soltani and M. H. Khooban, Fuzzy sliding mode control design for a class of nonlinear systems with structured and unstructured uncertainties, Int. J. Innov. Comput. Inform. Control 9 (2013), 2713-2726.

[39] M. W. Spong, S. Hutchinson and M. Vidyasagar, Robot Modeling and Control, 3, Wiley, New York, 2006.

[40] M. D. Tran and H. J. Kang, Adaptive fuzzy PID sliding mode controller of uncertain robotic manipulator, in Intelligent Computing Theories and Methodologies, pp. 92-103, Springer, Berlin, 2015.

[41] V. I. Utkin, Sliding Modes in Control and Optimization, Springer Science \& Business Media, 2013.

[42] V. Utkin, J. Guldner and J. Shi, Sliding Mode Control in Electro-Mechanical Systems, 34, CRC Press, Boca Raton, FL, 2009.

[43] S. T. Venkataraman and S. Gulati, Terminal sliding modes: a new approach to nonlinear control synthesis, in: Fifth International Conference on Advanced Robotics, 1991, 'Robots in Unstructured Environments', 91 ICAR, pp. 443-448, IEEE, 1991.

[44] B. Yao and M. Tomizuka, Adaptive robust motion and force tracking control of robot manipulators in contact with compliant surfaces with unknown stiffness, J. Dynam. Syst. Meas. Control 120 (1998), 232-240.

[45] X. Yu, Z. Man and B. Wu, Design of fuzzy sliding-mode control systems, Fuzzy Sets Syst. 95 (1998), 295-306.

[46] D. Zhao, S. Li, Q. Zhu and F. Gao, Robust finite-time control approach for robotic manipulators, IET Control Theory Appl. 4 (2010), 1-15.

[47] M. Zhihong, A. P. Paplinski and H. R. Wu, A robust MIMO terminal sliding mode control scheme for rigid robotic manipulators, IEEE Trans. Autom. Control 39 (1994), 2464-2469. 